\title{
Can Quantum Mechanics and Relativity be Considered Per Se Complete? - A discussion on Quantum Mechanics and Relativity in Full Space-time Domain

\author{
Yuanjie Li ${ }^{1}$, Lihong Zhang ${ }^{2}$, Peng Dong ${ }^{2 *}$
} \\ ${ }^{1}$ School of Optics and Electronic Information, Huazhong University of Science and Technology, Wuhan 430074, China \\ yuanjie_li@sina.com \\ ${ }^{2}$ Department of Fundamental Physics, College of Science, China University of Petroleum (East China), Qingdao 266580, China \\ zhanglh@upc.edu.cn \\ ${ }^{*}$ Corresponding to: dongpeng@upc.edu.cn
}

\section{ABSTRACT}

This paper points out the incompleteness of the traditional quantum mechanics and relativity, which is embodied in spacetime domains of definition, not in physical quantities for description. The real time and space are not continuous. The phenomena called "ghost-like long-range action" by Einstein in fact occur in the time discontinuity points, that is, Time Quantum Worm Holes put forward by Hawking. This paper also gives an essential difference between the macroscopic random motion and the microscopic random motion, which is critical for understanding wave-particle duality.

\section{KEYWORDS}

completeness; space-time discontinuity; principle of special relativity; TQWH; wave-particle duality.

PACS: 03.65.-w, 03.30.+p, 03.67.Mn

\section{ACADEMIC DISCIPLINE AND SUB-DISCIPLINES}

Physics, Quantum Mechanics, Relativity

\section{SUBJECT CLASSIFICATION}

Physics Subject Classification.

\section{INTRODUCTION}

In 1960s, American physicist Feynman said a famous saying: "I think I can safely say that nobody understands quantum mechanics." Half a century later, about what is wave-particle duality, what is Schrödinger cat, what is single photon interference, what is Einstein-Podolsky-Rosen (EPR) paradox, what is quantum entanglement and so on, no one throughout the world can explain clearly, even though they have already been confirmed in experiments. In 2005,125 questions that point to gaps in human's basic scientific knowledge were selected to celebrate Science's 125th anniversary. We believe that this paper provides an answer for one of these questions which is "Do Deeper Principles Underlie Quantum Uncertainty and Nonlocality?" [1]

It is well known that since the birth of relativity and quantum mechanics there has been being a fundamental contradiction: Einstein's theory of relativity asserts that the speed of motion of any matter in the universe has an upper limit, which is the speed of light; while quantum mechanics implies that the faster-than-light behaviors of microscopic particles are very common. A strange thing is that, about these behaviors, those who had ever made excellent contributions to quantum mechanics only emphasize whether results are correct or not, avoiding talking about processes and the mechanisms, in another word, they know what a thing is, but do not know why. In 1935, A. Einstein, B. Podolsky, and N. Rosen raised questions about quantum mechanics in their article titled "Can Quantum-Mechanical Description of Physical Reality Be Considered Complete?" [2], which is famous in the world and called EPR paradox. Einstein found the incompleteness of quantum mechanics; however he never thought of that the incompleteness of quantum mechanics results from the incompleteness of the theory of relativity. He mistakenly assumed that there might be lack of a hidden variable for description in quantum mechanics. Later, misdirected by Einstein, Bell proposed the Bell's inequality used to test whether quantum mechanics is complete or not in experiment [3]. Actually, the incompleteness of relativity and quantum mechanics does not lie in lack of variables describing them, but the incompleteness of space-time domain of definition. Briefly, the traditional theory of relativity and quantum mechanics are defined in measurable continuous spacetime domain, while immeasurable discontinuity points exist in the real space-time domain. The incompleteness of the theory of relativity and quantum mechanics just occurs at these space-time discontinuity points.

\section{MATHEMATICAL AND PHYSICAL CHARACTERISTICS OF DISCONTINUITY POINTS}

It's known that discontinuity points of mathematical functions include finite discontinuity points and infinite discontinuity points. Mutation of a function which occurs at a discontinuity point corresponds to phase transition in physics. If not only physical quantities but also the differentiation of them mutate at discontinuity points, the phase transition is called secondorder phase transition in physics. If there are discontinuity points in time, then we naturally wonder if there is any physical quantity mutating. In June 2015, we published an article entitled "A Supplement to the Invariance Principle of the Speed of 
Light and the Quantum Theory" [4]. The US Global Magazine made the following comment: "Your research is having a very good impact upcoming". In this article, we proposed that mutation happens in the speed of light, and it is an infinite discontinuity mutation. This is a second-order phase transition at time discontinuity points. It is very easy to prove this conclusion by applying the traditional uncertainty principle of quantum theory and the space-time Lorentz invariant of relativity.

We are here to thank the British physicist Hawking who proposed the existence of time discontinuity points and named them the Time Quantum Worm Holes, abbreviated as TQWHs. In the article [5], we made such a supplement to the invariance principle of the speed of light of relativity as follows: the speed of light $c$ is the upper limit of the speed of motion of cosmic matter, and remains constant in all inertial systems. $\mathrm{c}$ is a finite constant value in measurable observation time range, while it is infinitely large $(\mathrm{c}=\infty)$ at immeasurable observation time discontinuity points. Relativity becomes complete with such a supplement. Einstein no longer has to worry about that quantum mechanics will violate his theory of relativity.

\section{FASTER-THAN-LIGHT PHENOMENA IN QUANTUM MECHANICS CAN BE FOUND ANYWHERE WITHOUT TQWHS.}

The speed of light is infinitely large in TQWH, so for other particles with non-zero static mass, even if their speeds are infinitely large, they are still not larger than the speed of light. But if there is no TQWH, quantum mechanics cannot explain many problems as follows:

\subsection{One-dimensional infinitely deep well}

As shown in Fig.1, the probability density wave of a single electron in a one-dimensional infinitely deep well with a width of $40-50 \mathrm{~nm}$ is simulated [5]. Assume that positions where the electron appears with the largest probability are measured in a time error range of $10^{-17} \mathrm{~s}$, where the two positions are indicated by two black points connected by a red line in the figure; we can obtain two queries on quantum mechanics just by making simple estimates in order: (1) If the single electron goes from one of the two positions to the other in the observable time and space, its speed is

$$
v=\frac{20 \times 10^{-9} \mathrm{~m}}{10^{-17} \mathrm{~s}}=20 \times 10^{8} \mathrm{~m} / \mathrm{s}>c \text {. }
$$

It clearly violates the special theory of relativity; (2) As the single electron may travel back and forth between the two spatial positions with the largest probability for many times, in another word, as the single electron may pass through the central point with probability of zero for many times, obviously, it is impossible for the central point with probability of zero to exist as long as the speed of the single electron is finite, while the quantum mechanics accurately predicts that the probability of the center point is zero.

For the above two queries, we can only have two explanations. First, the single electron did definitely not pass in our observable time and space, there must exist a space-time blind channel, and the single electron passed through the unobservable space-time blind channel; second, the speed of the single electron in the space-time blind channel is infinitely large, and it almost does not need time to appear anywhere it may go at the same time, so the single particle becomes a wave. Where might the single particle go? It is the place where the principle of stability is met, that is, where the solution of the Schrödinger equation is satisfied. For one-dimensional infinitely deep well, it is the standing wave solution. The single electron passes through the TQWH at infinitely large speed, and almost simultaneously appears at the places of standing wave solution where the principle of stability is met, thereby achieving the physical process of transition from a particle to a wave. This is the essence of the wave-particle duality of quantum mechanics. Please watch the movie $\mathrm{S} 1$ to observe how a single particle in one-dimensional infinitely deep well changes into a wave.

\subsection{Schrödinger cat}

In 1991, an experiment on Schrödinger electronic cat was done successfully in a lab in Rochester University [6], in which an electron was found to appear simultaneously at two different places in a spatial distance of $\Delta x=10^{-7} \mathrm{~m}$. If we call the thing that the electron is in one of the two places as living state, symbolized by $|A\rangle$, and the thing that the electron is at the other place as dead state, symbolized by $|D\rangle$, then the electron is being at a superimposed state $\psi=\frac{\sqrt{2}}{2}|A\rangle+\frac{\sqrt{2}}{2}|D\rangle$. If we assume that the time measurement error is $10-17 \mathrm{~s}$, it is not difficult to calculate the speed of the electron running back and forth between the dead state and living state to be $10^{10} \mathrm{~m} / \mathrm{s} \square \mathrm{c}$. In fact, Schrödinger cat is the simplest superposition state of only two possible Eigen states, while the mathematical expression of the wave function of a single particle in quantum mechanics is a superposition state of infinite number of possible Eigen states. The wave function $\psi(\boldsymbol{r}, t)$ of a single particle indicates that the single particle can appear anywhere in the space at the same time $t=t_{0}$.

The Eigen state of the single particle appearing in space $\boldsymbol{r}_{\mathbf{i}}$ at time of $t=t_{0}$ is written as $P\left(\boldsymbol{r}_{i}, t_{0}\right)$, then

$$
\psi\left(\boldsymbol{r}, t_{0}\right)=\sum_{i} a_{i} P\left(\boldsymbol{r}_{i}, t_{0}\right)
$$


Quantum mechanics regards the Schrödinger cat as an exotic thing, while turns a blind eye to the much more complicated wave function of a single particle compared to the Schrödinger cat, which has to be surprised and amazing.

The above discussion allows us to believe firmly that any quantum phenomenon which is associated with wave function, if there is no TQWH, must imply faster-than-light behaviors. So the faster-than-light phenomena in quantum mechanics can be found anywhere without TQWH.

\section{MACROSCOPIC RANDOM MOTION AND MICROSCOPIC RANDOM MOTION}

Feynman thought that no one can understand quantum mechanics. The results of our study show which point of quantum mechanics is not understood and this point is just the essential difference between the macroscopic random motion and the microscopic random motion. Macroscopic randomness is a one-time behavior of multiple particles or multiple behaviors of a single particle. In the study of the motion of gas molecules, the "multiple" means the order of magnitude of the Avogadro constant, $10^{23}$. Moreover, the macroscopic randomness is the randomness of initial conditions and boundary conditions; as far as the motion of an individual particle, it follows deterministic theory. However, the microscopic quantum randomness is a one-time behavior of a single particle. The so-called randomness actually means that the development of things will result in a number of possible results. In the macroscopic randomness, a single particle can only take one of the possible results for one time, while, for microscopic quantum randomness, a single particle can take all the possible results simultaneously for one time. To achieve this, a microscopic quantum must meet two conditions: (1) there exist space-time blind channels; (2) it has infinitely large speed. Complete relativity and quantum mechanics just support these conditions. Once you understand the essential difference between the macroscopic and microscopic random motions, the emergence of quantum entangled state is not out of expectation.

The phenomena which were called "ghost-like long-range actions" by Einstein are quite normal in the TQWHs. Infinite time discontinuity points or TQWHs are distributed compactly on time axis, just like irrational numbers are distributed compactly on real number axis. The speed of light at the time discontinuity points or TQWHs has been mutated to infinity, so for the speed of any other particles with non-zero static mass, even if it is infinitely large, it is still no larger than the speed of light. This is the conclusion of a complete theory of relativity, which provides quantum mechanics with magical space-time blind channels, and all "ghost-like long-range actions" have become normal phenomena. Since then the quantum mechanics is also complete. The last-for-a-century quarrel between quantum mechanics and relativity will end after the publication of this article, and from then on, it will be a new era with quantum mechanics and relativity being brought into harmony. Truth is simple and beautiful.

\section{FIGURES/CAPTIONS}

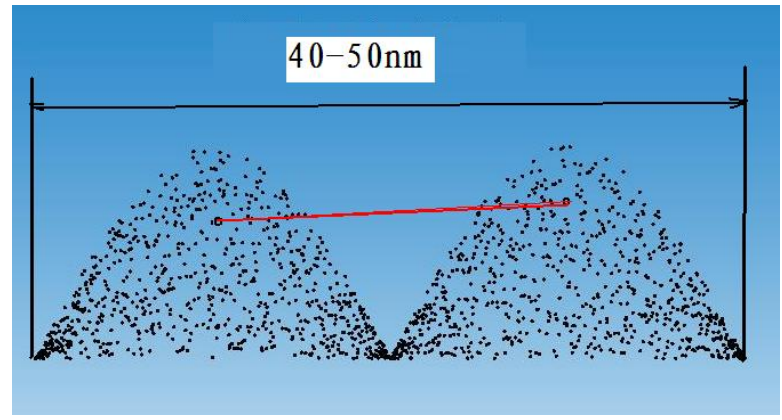

Fig. 1 The probability density wave of a single electron in a one-dimensional infinitely deep well

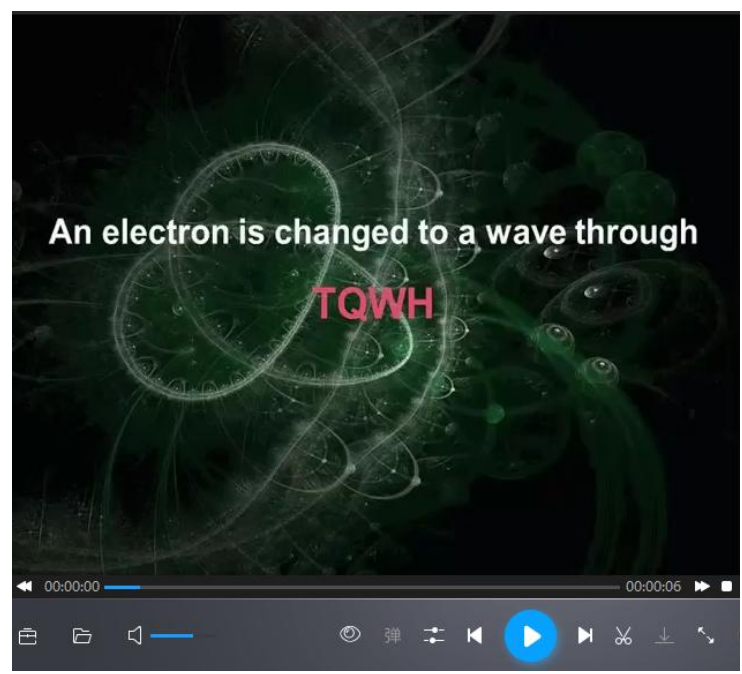

Video S1 An electron is changed to a wave through TQWH. 


\section{REFERENCES}

1. Charles Seife. Do deeper principles underlie quantum uncertainty and nonlocality? Science, 2005, 309, 98.

2. Einstein, B. Podolsky, and N. Rosen. Can quantum-mechanical description of physical reality be considered complete? Physic Review, 1935, 47, 777-780.

3. J. S. Bell. On the problem of hidden variables in quantum mechanics. Reviews of Modern Physics, 1966, 38, 447-452.

4. Y. Li, W. Jia, J. Wang. A supplement to the invariance principle of the speed of light and the quantum. Jurnal of Modern Physics, 2015, 6, 126-130.

5. G. Engels, J. Lange, Th. Schapers, and H. Luth. Experimental and Theoretical approach to spin splitting in modulation-doped InxGa1-xAs/InP quantum wells for B $\rightarrow 0$. Physics Review B, 1997, 55, R1958-1961.

6. John A.Yeazell , C. R.Stroud, Jr. Observation of fractional revivals in the evolution of a Rydberg atomic wave packet. Physics Review A, 1991,43, 5153-5156.

\section{Author' biography with Photo}

Yuanjie $\mathrm{Li}$ is a professor of Huazhong University of Science and Technology, Wuhan, China. His research work forcuses on theoretical physics about Quantum mechanics, Relativity, Dark matter and Dark energy etc.

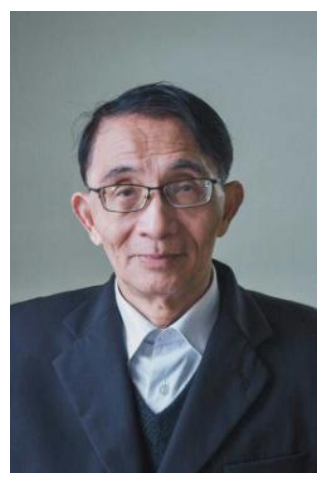

Lihong Zhang is an associate professor of China Univeristy of Petroleum(East China), Qingdao, China. She focuses her work on university physics teaching. In recent years, she is also interested in research work on Quantum mechanics, Relativity etc.

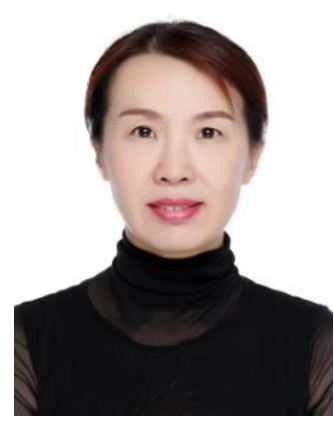

Peng Dong is a lecturer of China University of Petroleum(East China), Qingdao, China. Her research interests include nonlinear optical effects in fibers, supercontinuum generation in fibers, nonlinear optical effects in high power fiber lasers and so on. She is also interested in research works on Quantum Mechanics in recently years.

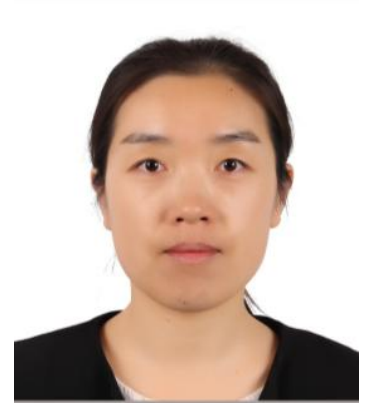


I S S N $2347-3487$

Volume $14 \mathrm{Number} 1$

Journal of Advances in Physics 